\title{
Graz Brain-Computer Interface (BCI) II $^{1}$
}

\author{
J. Kalcher, D. Flotzinger, S. Gölly, Ch. Neuper, G. Pfurtscheller \\ Institute of Biomedical Engineering, Department of Medical Informatics, \\ and Ludwig Boltzmann-Institute of Medical Informatics and Neuroinformatics, \\ Graz University of Technology, Brockmanngasse 41, A-8010 Graz/Austria
}

\begin{abstract}
This paper describes the new setup of the Graz Brain-Computer Interface (BCI) system $\mathrm{II}$, which is based on on-line classification of EEG patterns to determine which of three kinds of movement is planned by a subject. This classification can be exploited for on-line control which may constitute a great help for handicapped persons in the future.
\end{abstract}

\section{Introduction}

Theoretically, the thoughts of a person should be reflected in the person's brainwaves and therefore be measurable by electrodes on the person's scalp. In practice, several groups have shown that such 'thoughts' as mentally answering 'yes' and 'no' [1], intention to move a joystick [2] or planning of hand movement $[3,4]$ can be discriminated based on the recorded EEG with surprising accuracy. These findings have led to the idea to exploit the EEG for control in cases where other means of control are either impossible, e.g. in cases of handicapped persons, or infeasible, e.g. if both hands are occupied.

A system which uses EEG to build a communication line between the brain and an electrical appliance has become known as a Brain-Computer Interface (BCI). Several teams all over the world are currently working at such systems $[5,6]$; this paper describes the work done in Graz, a system which is mainly based on the discrimination of various types of movement planning $[7,8]$.

\section{Experimental Paradigm}

The basic idea of the Graz BCI system has already been outlined in previous papers[7]. The Graz BCI I was a one-dimensional cursor control system which could discriminate between left and right hand movement planning whereby in the initial session the subject had to press a microswitch with either the left or right index finger. The cortical areas involved in these two kinds of movement planning are primarily the left and right sensorimotor hand areas; the corresponding electrode positions overlying these areas are $C_{3}$ and $C_{4}$ (international 10-20 system) [3].

\footnotetext{
1 Supported by the "Fonds zur Förderung der wissenschaftlichen Forschung", project P9043 and "Forschungsförderungsfonds für die gewerbliche Wirtschaft" project $2 / 312$.
} 
To increase the dimensions of control, additional EEG patterns have to be found which are discernable from left and right hand movement. Studies of foot and tongue movement showed that these two kinds of movement can indeed be discriminated from left and right hand movement, especially in the movement planning phase [8] (see Fig. 1). Therefore, the additional movernent type 'foot flexion' was built into the Graz BCI which, due to several changes in the recording scheme and experimental paradigm, is now called Graz BCI II.

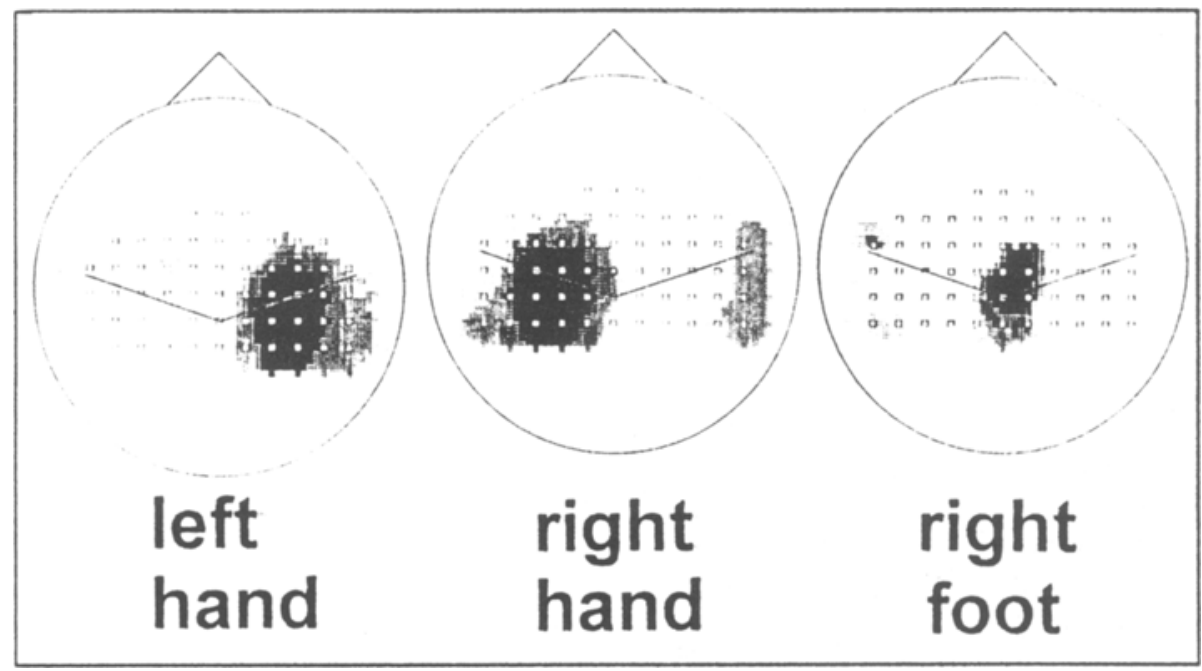

Fig. 1. Topographic maps display alpha power decrease during planning of hand and foot movement. "Black" indicates cortical areas with large power decrease. The electrode positions and the approximate location of the central sulcus are indicated.

The basics of a trial of the Graz BCI II are shown in Fig. 2. The subject is seated in a comfortable chair looking at a fixation cross on a monitor 1 meter in front of the subject's eyes. One second after an acoustic warning stimulus ('beep') a cue in the form of an arrow, pointing either left, right or down. appears and indicates to the subject that as soon as the arrow vanishes (after $1250 \mathrm{msec}$ ) he should either press a microswitch with his left index finger, his right index finger or move the toes of his right foot upwards (dorsal flexion), respectively. A 1-second period starting 250 msec after presentation onset of the cue is classified into one of the three movement tasks and the corresponding classification of the system is fed back to the subject either as correctly identified $\left({ }^{\prime}+\right.$ '), undecided $\left({ }^{\prime}\left({ }^{\prime}\right)\right.$ or wrong classification $\left({ }^{\prime}-'\right)$. 


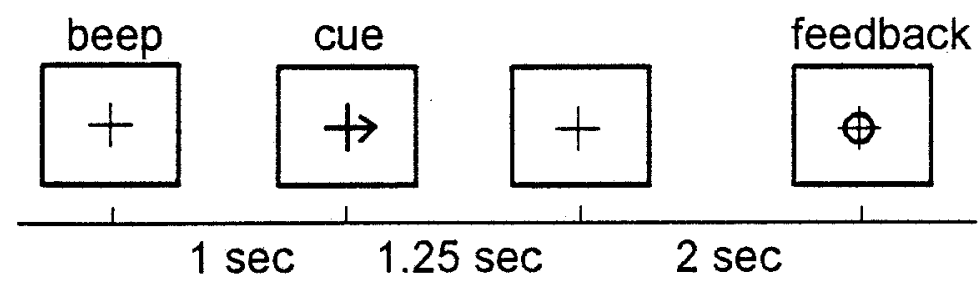

Fig. 2. Experimental paradigm of the Graz BCI II

A PC486 with a DSP-board records and classifies the EEG and provides the feedback to the subject. Three bipolar EEG channels are used for on-line classification: $\mathrm{C}_{3}-\mathrm{C}_{3}^{\prime}, \mathrm{C}_{2}-\mathrm{C}_{2}^{\prime}$ and $\mathrm{C}_{4}-\mathrm{C}_{4}^{\prime}$ (see Fig. 2). The signals are sampled at 64 $\mathrm{Hz}$, whereby the features extracted from the 1 -second period of $E E G$ and presented to the classifier are comprised of four power estimates, each representing $250 \mathrm{msec}$, per EEG channel. These power estimates are calculated by squaring each sample and then averaging over 16 samples. The 12 features ( 3 EEG channels times 4 power estimates) are offered to a classifier which calculates both a classification and a measure describing the certainty with which the classification was obtained. Depending on this quality measure and the correctness of the classification, the system provides a feedback to the subject in the form of a small or large ' + ' if the classification is correct and the quality measure is in medium or large range, respectively, a small or large ' - ' if the classification is incorrect, and ' $O$ ' if the quality measure is very small.

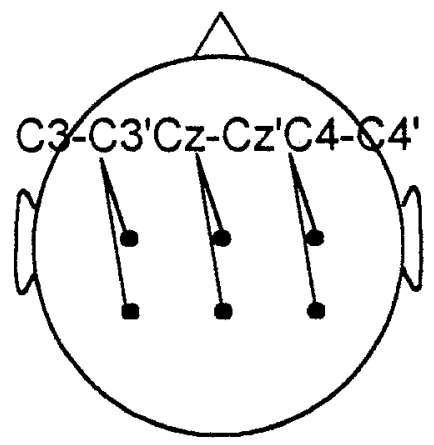

Fig. 3. Positions of the electrodes on the scalp

Four subjects ( 1 female and 3 male students aged between 23 and 27 years) participated in four experimental sessions on different days within 2 weeks. Each session lasted about $1.5 \mathrm{~h}$ and comprised four blocks of 60 trials each with 5 min breaks between blocks. In the first session, data were collected for the creation of the classifier and therefore no feedback was provided. In the following sessions 2 and 3 , the classifier was used to discriminate the 3 kinds of movement out of the movement 
planning phase and to give feedback to the subject as described above. In session 4 no physical behavior took place, using the same stimulation procedure as in the first sessions, the subject was asked to concentrate on left hand, right hand or foot movement. In this session the classifier was applied to EEG data during mental activity and provided feedback about discriminability of three different mental states.

\section{Classification Method}

The classifier built into the Graz BCI II system is a Learning Vector Quantizer (LVQ) $[9,10]$. Its classification speed and ease of use in the Graz BCI I system have suggested its further use in on-line EEG classification of more than two categories.

Basically, a LVQ is a nearest-neighbour classifier whereby a labelled codebook is generated such that for each training example the nearest codebook vector is of the same category as the given example. Thereby, LVQ tries to optimize the representation of the training examples by the codebook vectors (also known as Vector Quantization) and at the same time to minimize the classification error. The labelled codebook is generated using an iterative learning procedure where in each iteration a randomly selected training example of known classification is compared to the codebook and the two closest codebook vectors are found. Depending on the class labels of the given example and of the two codebook vectors the latter two are updated by either pushing them towards the example (if the class labels are identical to the known classification of the example, correct classification) or pushing them away from the example (for a detailed description of the algorithm see $[9,10]$. The overall aim of this learning algorithm is to increase the probability of correct classification the next time the given example is presented.

Extensive off-line analyses have shown that only a very small number of codebook vectors is needed to provide satisfactory performance. usually $3-4$ codebook vectors per category suffice. Searching twelve (4 codebook vectors times 3 categories) 12-dimensional vectors for the nearest codebook vector is an extremely simple and fast form of classification.

The quality measure of the classification can be obtained by not only searching for the nearest codebook vector but also for the nearest codebook vector of a second category. By comparing the distances of these two codebook vectors it can be determined whether the given example lies near a class boundary, in which case the distances to the two codebook vectors $d_{1}$ and $d_{2}$ will be very similar, or whether the given example clearly belongs to the category of the closest codebook vector, in which case distance $d_{1}$ will be much smaller than $d_{2}$. Therefore, the quality measure can be computed as

$$
\operatorname{qu}\left(d_{1}, d_{2}\right)=1-\frac{d_{1}}{d_{2}} .
$$

It is obvious that this formula will provide values close to zero if the distances $d_{1}$ and $d_{2}$ are about the same and values close to one if distance $d_{1}$ is much smaller than $d_{2}$. 


\begin{tabular}{|c|c|c|c|c|c|}
\hline 'A6' & big + & + & 0 & - & big - \\
\hline Session 2 & $21,7 \%$ & $37,5 \%$ & $21,7 \%$ & $16,7 \%$ & $2,5 \%$ \\
\hline Session 3 & $25,0 \%$ & $34,6 \%$ & $23,8 \%$ & $13,3 \%$ & $3,3 \%$ \\
\hline Session 4 & $20,8 \%$ & $24,2 \%$ & $24,2 \%$ & $24,6 \%$ & $6,3 \%$ \\
\hline
\end{tabular}

\begin{tabular}{|c|c|c|c|c|c|}
\hline 'B9' & big + & + & 0 & - & big - \\
\hline Session 2 & $20,0 \%$ & $21,7 \%$ & $15,4 \%$ & $30,4 \%$ & $12,5 \%$ \\
\hline Session 3 & $18,8 \%$ & $23,3 \%$ & $15,8 \%$ & $28,8 \%$ & $13,3 \%$ \\
\hline Session 4 & $12,9 \%$ & $20,8 \%$ & $22,5 \%$ & $28,8 \%$ & $15,0 \%$ \\
\hline
\end{tabular}

\begin{tabular}{|c|c|c|c|c|c|}
\hline 'Al' & big + & + & 0 & - & big - \\
\hline Session 2 & $22,9 \%$ & $27,5 \%$ & $18,8 \%$ & $23,3 \%$ & $7,5 \%$ \\
\hline Session 3 & $20,0 \%$ & $21,3 \%$ & $20,8 \%$ & $26,7 \%$ & $11,3 \%$ \\
\hline Session 4 & $19,6 \%$ & $24,2 \%$ & $20,4 \%$ & $21,3 \%$ & $14,6 \%$ \\
\hline
\end{tabular}

\begin{tabular}{|c|c|c|c|c|c|}
\hline 'B8' & big + & + & 0 & - & big - \\
\hline Session 2 & $10,4 \%$ & $27,9 \%$ & $21,7 \%$ & $33,3 \%$ & $6,7 \%$ \\
\hline Session 3 & $21,7 \%$ & $25,0 \%$ & $13,8 \%$ & $29,6 \%$ & $10,0 \%$ \\
\hline Session 4 & $14,2 \%$ & $26,3 \%$ & $24,6 \%$ & $26,3 \%$ & $8.8 \%$ \\
\hline
\end{tabular}

Table 1. On-line performance (\%) of four subjects in three sessions.

\section{Results and Discussion}

The on-line performance for the last three sessions (the first was used for training the classifier) of each subject are given in Table 1. All subjects showed more than random on-line performance (random performance in 3 categories would be 33,33\% correct).

From Table 1 can be seen:

(i) There are always more trials classified correct $(+)$ compared with incorrect (-). 
(ii) About $20 \%$ of the trials are not classified into any of the 3 classes. This number depends on the threshold used and is a potential for further improvement.

(iii)In the first "mental" session (session 4 ) for three subjects the majority of trials were correctly classified

These results are far from optimal but they show for the first time that after only 4 sessions a discrimination between 3 different EEG patterns is possible. It has to be kept in mind, that the systems of Clark and Tizard [1], Hiraiwa et al. [2] and Wolpaw et al. [5] were all designed for a 2-class discrimination problem and none of these systems were applied to 3 classes.

There are different possibilities for further improvement:

(i) Selection of the optimal frequency band with the largest EEG reactivity. For the parameter estimation not only the alpha but also the beta band can be used.

(ii) Selection of the optimal electrode positions. It was shown recently, that classification results depend on the location of the electrodes [11] whereby the optimal positions are not the same for each subject. Such electrode positions should be selected in a pre-experiment, where the EEG is recorded from a large number of electrodes.

(iii) Increase the number of sessions.

\section{Conclusion}

The design of the Graz BCI is based on mental preparation of different kinds of movement. It was shown, that 3 "movement patterns" can be differentiated in an online experiment within fractions of a second. It is planned to extend the Graz BCI to 4 classes, whereby the 4 th class can be e.g. visual imagination.

There are, however, still a number of problems to be solved in relation to optimize the performance of the system. Among these are the improvements in the experimental paradigm including a more effective feedback, the estimation of more specific EEG parameters and improvements in the learnable classifier. As already mentioned, a very important task is also the selection of the optimal electrode positions.

\section{References}

1. Clark C.R., Tizard J.: Single-trial analysis of scalp electrical fields using artificial neural networks, Proceedings of 3rd Int. Congress on BET Amsterdam, 1992.

2. Hiraiwa A., Shimohara K., Tokunaga Y.: EEG topography recognition by Neural Networks, Engineering in Medicine and Biology, pp. 39-42, 1990. 
3. Pfurtscheller G., Berghold A.: Patterns of cortical activation during planning of voluntary movement, Electroenceph. and clin. Neurophysiol., Vol. 72, pp. 250$258,1989$.

4. Pfurtscheller G., Flotzinger D., Mohl W., Peltoranta M.: Prediction of the side of hand movements from single-trial multi-channel EEG data using neural networks, Electroenceph. and clin. Neurophysiol., Vol. 82, pp. 313-315, 1992.

5. Wolpaw J. R., McFarland D., Neat G. W., Forneris C. A.: An EEG-based braincomputer interface for cursor control, Electroenceph. and clin. Neurophysiol., Vol. 78, pp. 252-259, 1991.

6. McFarland D. J., Neat G. W., Read R. F., Wolpaw J. R.: An EEG-based method for graded cursor control, Psychobiology, Vol. 21, No. 1, pp. 77-81, 1993.

7. Pfurtscheller G., Flotzinger D., Kalcher J.: Brain-Computer Interface - a new communication device for handicapped persons, Journal of Microcomputer Appl., Vol. 16, pp. 293-299, 1993.

8. Pfurtscheller G., Flotzinger D., Neuper Ch.: Differentiation between finger, toe and tongue movement in man based on $40-\mathrm{Hz}$ EEG. Electroenceph. and clin. Neurophysiol, Vol. 90, pp. 456-460, 1994.

9. Kohonen T.: The Self-Organizing Map, Proceedings of the IEEE, Vol. 78, No. 9 , pp. 1464-1480, 1990.

10. Flotzinger D., Kalcher J., Pfurtscheller G.: EEG Classification by Learning Vector Quantization, Biomedizinische Technik, Vol. 37, No. 12, pp. 303-309, 1992.

11. Flotzinger, D., Pregenzer, M., Pfurtscheller, G.: Feature Selection with Distinction Sensitive Learning Vector Quantisation and Genetic Algorithm Proceedings of IEEE conference on Neural Networks (ICNN 94), pp. $3448-3451$, 1994. 